vorlesung auf dem Freiburger Soziologiekongress von 1998, die nunmehr auch in übertragenem Sinne in den Rang einer Verabschiedung rückt. Unter dem Titel „Die Kreativität religiöser Ideen“ galt sie dem Versuch, die „Frage nach den anthropologischen Grundlagen religiöser Ideen zu beantworten". Den Status eines Vermächtnisses kann sie nicht nur insofern beanspruchen, als sie Popitz' anthropologisch orientiertes Nachdenken auf Kernphänomene von Sinnstiftungsprozessen zuspitzt, sondern auch, weil damit die ebenso die Baseler Antrittsvorlesung wie auch die Überlegungen zur Entwicklungsgeschichte technischen Handelns anleitende Frage nach der ,sozialen Produktivität des Menschen" eine systematische Abrundung erfährt. Wobei Popitz erneut - wie schon in seinen Arbeiten über Macht, Recht und Technik - den Blick nicht zuletzt auf die Ambivalenzen menschlichen Lebens „zwischen Entfremdung und Verwirklichung" richtet.

$\mathrm{Zu}$ Popitz' Grundhaltungen gehörte die beständige kritische Distanz zum eigenen Denken und Forschen. Einer seiner von Ralf Dahrendorf zur Kenntnis gebrachten Schüttelreime bringt die daraus resultierende Haltung der Offenheit wie Grundsätzlichkeit seines Fragens schön zum Ausdruck, wenn es heißt: „In der Erkenntnis weitem Zelt / durchforschest du die Zeitenwelt / und suchest nach dem Immer-Gleichen, / willst auch den kleinsten Glimmer eichen - / und find'st doch nur die Fabelnorm / in deiner eignen Nabelform." Heinrich Popitz, der den Erkundungen des allgemeinen menschlichen Erfahrungs- und Gestaltungspotenzials seine intellektuelle Energie widmete, ist am 1. April 2002 verstorben.

Martin Endreß

\section{Berlin Graduate School of Social Sciences}

Das Institut für Sozialwissenschaften der Humboldt-Universität richtet ab Herbst eine „Berlin Graduate School of Social Sciences“ ein, die aus Programmen des DAAD und der DFG gefördert wird. Die BGSS baut auf dem neuen BA/MA-Studiengang, der $a b$ dem Wintersemester 2002/2003 an die Stelle des alten Diplomstudiengangs Sozialwissenschaften treten wird, und den bereits bestehenden internationalen Programmen -- dem „Euromasters", dem „Trans-Atlantic Masters“ und dem Graduiertenkolleg „Das neue Europa“ auf. Sieben bis acht neue Kollegiaten aus dem In- und Ausland werden zu uns stoßen und ihre Dissertationen in einem der vier Arbeitsschwerpunkte des Instituts verfolgen. Der Schwerpunkt „Europäische Studien“ bildet dabei das Startmodul. Hinzu kommen „Vergleichende Kulturund Institutionenanalyse", „Zivilgesellschaft und Demokratie" sowie "Arbeit und Lebensführung". Die BGSS wird vom Institut für Sozialwissenschaften und seinem gesamten wissenschaftlichen Personal mitgetragen. Das Institut verfügt über eine professionelle Infrastruktur im Herzen Berlins. Zusätzlich werden wir in- und ausländische Gastprofessoren gewinnen, die die Lehre bereichern sollen. Die BGSS wird sich mit einschlägigen Einrichtungen weltweit vernetzen, um den internationalen Austausch für den angehenden sozialwissenschaftlichen Nachwuchs zu optimieren. Webseite und Internet-Auftritt sind in Arbeit. Interessierte wenden sich bitte für nähere Auskünfte und Informationen an Martin Nagelschmidt (Martin.Nagelschmidt@rz.hu-berlin.de) für das Promotionsprogramm und an Kathleen Shanahan für die Masters-Studiengänge (Kathleen.Shanahan@rz.hu-berlin.de).

Hans-Peter Müller

\section{Preis der Fritz Thyssen Stiftung für sozialwissenschaftliche Aufsätze des Zeitschriftenjahrgangs 2001}

Zum einundzwanzigsten Mal wurden durch eine Jury, die im Institut für Angewandte Sozialforschung der Universität zu Köln tagte, die Preise der Fritz Thyssen Stiftung für die besten sozialwissenschaftlichen Aufsätze in deutscher Sprache vergeben. Dies ist der einzige Zeitschriftenpreis in den Sozialwissenschaften außerhalb des englischsprachigen Bereichs.

Die Auswahl der Arbeiten erfolgt in zwei Stufen. Die Herausgeber und Redakteure von dreizehn deutschsprachigen Zeitschriften schlagen jeweils bis zu zwei Aufsätze vor, die anschließend von einer Jury begutachtet werden. Die am Verfahren beteiligten Zeitschriften sind:

Angewandte Sozialforschung,

Berliner Journal für Soziologie, Geschichte und Gesellschaft, Kölner Zeitschrift für Soziologie und Sozialpsychologie,

Leviathan, 